(C) [2006] IEEE. Reprinted, with permission, from [R. C. Hoft, M. J. Ford and M. B. Cortie, Effect of dipole moment on current-voltage characteristics of single molecules, Nanoscience and Nanotechnology, 2006. ICONN '06.

International Conference on 3-7 July 2006]. This material is posted here with permission of the IEEE. Such permission of the IEEE does not in any way imply IEEE endorsement of any of the University of Technology, Sydney's products or services. Internal or personal use of this material is permitted. However, permission to reprint/republish this material for advertising or promotional purposes or for creating new collective works for resale or redistribution must be obtained from the IEEE by writing to pubs-permissions@ieee.org. By choosing to view this document, you agree to all provisions of the copyright laws protecting it 


\title{
Effect of dipole moment on current-voltage characteristics of single molecules
}

\author{
R. C. Hoft, M. J. Ford and M. B. Cortie \\ Institute for Nanoscale Technology \\ University of Technology, Sydney, PO Box 123, Broadway NSW 2007, Australia \\ Email: mike.ford@uts.edu.au
}

\begin{abstract}
We perform empirical calculations of the tunneling current through various small organic molecules sandwiched between gold electrodes by using the Wenzel-KramersBrillouin (WKB) approximation. The barrier to tunneling is taken to be the work function of gold and calculated from a first principles electronic structure code. The current-voltage characteristics of these molecules are compared in the context of existing first principles and experimental results. In this model the surface dipole moment, induced by the adsorbed molecule, can have a significant effect on the current and hence dipole moments may be an important property for prediction of the conductance characteristics of a molecule.
\end{abstract}

Keywords- Molecular electronics, single molecule conductance, electron transport, ab-initio transport calculations, surface dipole moment.

\section{INTRODUCTION}

Studies of electron tunneling between two electrodes with adsorbed bridging molecules are attracting considerable attention due to the possible application of this configuration in the fabrication of an electronic device with components consisting of molecules [1]. The response of such a system to an applied bias between the electrodes depends on the geometric and electronic structure of the molecule, the coupling between the molecule and electrodes and the potential profile across the molecule.

The most prolific experimental technique used to probe the current-voltage characteristics of electrode-moleculeelectrode systems is scanning tunneling microscopy (STM) [2-10]. An STM tip acting as top electrode is lowered onto a self-assembled monolayer of the molecule under study, which is attached to the bottom electrode. Thiolate molecules on gold surfaces are the most commonly studied system [11]. These scanning probe techniques suffer from large uncertainties associated with the nature of the coupling between the STM tip and molecule. Break junction experiments attempt to improve on this by creating a junction in which assembled molecules are chemically bound to both electrodes [12-14].

Ab-initio theoretical studies of the current-voltage characteristics that take into account a full quantummechanical description of the electrode- molecule -electrodesystem under an applied bias $[15,16]$ typically overestimate the experimental conductance of individual molecules $[2,3$,
$13,17]$ by two orders of magnitude. The contact geometries assumed in these ab-initio calculations are idealised and studies have been done to measure the actual effect that geometry variation has on the results [18-23]. While the uncertain experimental geometries could account for the large discrepancies between theory and experiment, other possible problems with the application of the most commonly used ab-initio technique, Density Functional Theory (DFT) $[24,25]$, to the transport problem have been pointed out by various authors [26-29].

To obtain a qualitative understanding of the transport in these systems it may be useful to simply describe the tunneling due to a barrier occurring in the gap between the top and bottom electrodes. The presence of a molecule will then affect the barrier shape and height. In this paper we investigate tunneling between two $\mathrm{Au}(111)$ electrodes in the presence of representative aromatic dithiols. We use the potential from an ab-initio electronic description of the gold slab with adsorbed molecule to obtain the barrier height for an electron to escape from the slab. We describe how this is related to the work function of gold and the surface induced dipole moment. The WKB approximation is then used to calculate corresponding current-voltage characteristics and this is compared with $a b$-initio $I(V)$ data.

\section{METHOD}

The optimum adsorption geometry and corresponding electronic structure of the molecules on an $\mathrm{Au}(111)$ slab was calculated with the SIESTA [30, 31] software package. Atom-centered basis functions are used for the valence electrons with a double zeta plus polarization orbital for each atom. The orbitals are strictly localized with the localization defined by an energy parameter, being the excitation energy due to the confinement. This parameter is set to $5 \mathrm{mRy}$. Core electrons are modeled with norm-conserving pseudopotentials constructed according to the scheme of Troullier and Martins [32]. The exchange and correlation energy is calculated with the generalised gradient approximation with parametrisation due to Perdew, Burke and Ernzerhof [33]. Conjugate gradient optimisations and surface scans are performed to find the minimum interaction energy of the molecules on an $\mathrm{Au}(111)$ surface approximated by 4 layers of $3 \times 3 \mathrm{Au}$ atoms repeated periodically in the plane parallel to the slab. 
The electronic structure of the optimum geometry is used to calculate the potential outside the slab and the bulk and slab Fermi energies. The surface dipole moment is calculated by integrating the charge from the middle of the slab outward to a position beyond the molecule.

We model tunneling through the gap with the WKB approximation for the transmission function $T(E)$ between electrodes

$$
T(E, V, d)=e^{-\frac{2 \sqrt{2}}{\hbar} \int_{0}^{d} \sqrt{m(U(x)-E)} d x}
$$

where $m$ is the electron mass, $d$ is the width of the barrier and $U(x)$ is the potential function across the barrier. The current is then found from the Landauer formalism as

$$
\begin{aligned}
i(V, d)=\frac{2 e}{h} \int_{-\infty}^{\infty} T(E, V, & d) \rho_{1}(E-e V) \rho_{2}(E) \\
\times & {[f(E)-f(E-e V)] d E }
\end{aligned}
$$

where $\rho_{1}$ and $\rho_{2}$ are the local densities of states of the top and bottom electrodes, $f(E)$ and $f(E-e V)$ are the Fermi-Dirac distribution functions for the two electrodes, and $V=V_{\text {top }}-$ $V_{\text {bottom }}$ is the bias applied between the electrodes with the convention that the top electrode is grounded. The contact conductance is taken to be the quantum of conductance, $2 e / h \approx 77 \mu \mathrm{S}$ [34]. We use the zero temperature approximation and assume a constant density of states to find the simplified relation

$$
i(V, d)=\frac{2 e}{h} \int_{0}^{e V} e^{-\frac{2 \sqrt{2}}{\hbar} \int_{0}^{d} \sqrt{m(U(x)-E} d x} d E
$$

We use a trapezoidal barrier with barrier height $\phi$ modified by a sinusoidal function which approximately accounts for image charging [35]

$$
U(x)=e V\left(1-\frac{x}{d}\right)+\phi\left[\sin \left(\frac{\pi x}{d}\right)\right]^{\alpha}
$$

with the dimensionless parameter $\alpha=0.2$ that controls barrier shape.

The barrier height is calculated using two methods: (A), the Fermi level of bulk gold modified by the energy required to move an electron through the induced surface dipole in the presence of the adsorbed molecule

$$
\phi_{1}=-E_{F, b u l k}+W_{\mu}
$$

This energy, $W_{\mu}$, is calculated from the dipole moment per unit area, $P_{o}$, by a simple parallel plate approximation,

$$
W_{\mu}=\frac{e P_{\sigma}}{\varepsilon_{0}}
$$

where $\varepsilon_{0}$ is the permittivity of vacuum. The dipole moment is extracted from the SIESTA DFT calculations as described above. In method (B), the difference in potential energy between the vacuum and the Fermi level of the surface including the effect of the adsorbed molecule is calculated directly in SIESTA. This quantity is well approximated by the difference in energy between the Fermi energy and potential calculated a large distance from the slab surface. In our case we take the potential in the middle of the gap between periodically repeating slabs.

$$
\phi_{2}=V_{v a c}-E_{F, \text { surface }}
$$

The barrier width $d$ is the distance between the two electrodes, taken as $9 \AA$ - this is roughly the length of all three molecules.

The DFT transport calculations are performed with the TRANSIESTA-C package [36]. The electronic structure is calculated according to the SIESTA method although we use a relaxed set of parameters which is sufficient for qualitative information. The transport is calculated using the nonequilibrium Green's functions method (NEGF). The device region is modeled as the molecule plus two layers of $\mathrm{Au}(111)$ on either side and is coupled to semi-infinite bulk electrode regions on the left and right. Including three $\mathrm{Au}(111)$ layers on either side of the molecule made no difference to the result of a test case.

\section{RESULTS AND DISCUSSION}

The analysis is carried out for the adsorbed molecules 1,4-dimethanethiol (XYL), ethynylbenzene (EB) and 1ethynyl-4-nitrobenzene (ENB) shown in Fig. 1.

The bulk Fermi level is calculated as $E_{F, b u l k}=3.01 \mathrm{eV}$. Table 1 lists the barrier heights calculated according to equations (5) and (7) for the bare gold slab as well as with each adsorbed molecule. Clearly the two methods result in the same trend for the barrier height for the different molecules and bare slab. Adsorption of XYL and EB reduce the surface dipole moment and hence the barrier height whereas ENB increases the dipole moment and hence barrier height. The parallel plate approximation (equation (5)) seems to overestimate the effect of the dipole moment on the work function (barrier height).

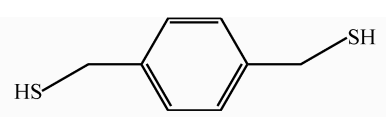

(a)

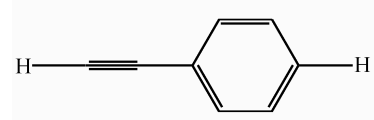

(b)

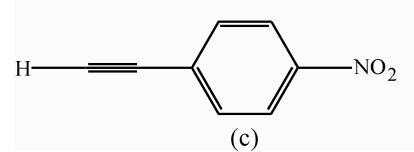

Figure 1. The molecules used in the investigation (a) 1,4-dimethanthiol (XYL), (b) ethynylbenzene (EB) and (c) 1-ethynyl-4-nitrobenzene (ENB). 
TABLE I. SURFACE DIPOLE MOMENT PER UNIT AREA $\mathrm{P}_{\sigma}$, ENERGY REQUIRED TO MOVE EN ELECTRON THROUGH THE DIPOLE $\mathrm{W}_{\mu}$, AND BARRIER HEIGHTS CALCULATED ACCORDING TO EQUATION (5) $\phi_{1}$, AND EQUATION (7) $\phi_{2}$ FOR THE MOLECULES IN FIG. 1.

\begin{tabular}{ccccc}
\hline \hline Molecule & $\begin{array}{c}P_{\sigma} \\
\left(10^{-2} \mathrm{e} / \mathrm{a}_{0}\right)\end{array}$ & $\mathrm{W}_{\mu}(\mathrm{eV})$ & $\phi_{1}(\mathrm{eV})$ & $\phi_{2}(\mathrm{eV})$ \\
\hline $\mathrm{Au}(111)$ & -1.14 & 3.90 & 6.91 & 4.57 \\
$\mathrm{XYL}$ & -0.97 & 3.31 & 6.32 & 4.42 \\
$\mathrm{~EB}$ & -0.78 & 2.66 & 5.67 & 4.27 \\
$\mathrm{ENB}$ & -1.60 & 5.47 & 8.48 & 5.09 \\
\hline \hline
\end{tabular}

Fig. 2 shows the corresponding current-voltage characteristics for both methods of calculating the barrier height. Although the absolute values are not of particular significance, we note that they are closer to recent state of the art experimental data than typical ab-initio calculations. Akkerman et. al. [37] recently obtained reliable measurements of the current through large-area molecular junctions and the current normalised to a single decanethiol molecule amounts to $\sim 0.01 \mathrm{pA}$. In contrast DFT calculations result in currents several orders of magnitude higher. Fig. 3 shows our DFT+NEGF results for the XYL, 1,4-diethynylbezene (DEB) and ENB molecules.

While conclusive experimental proof of differences in conductance between distinct molecules is rare, the conventional wisdom is that conductance is increased by small HOMO-LUMO gaps and a high level of conjugation of the molecules' orbitals. However recent STM break junction experiments by Tao et. al. [2, 3] that probe the conductance of aromatic- and alkane-dithiols do not support this. In fact they find a higher conductance for the alkane chains with more localized orbitals [22].

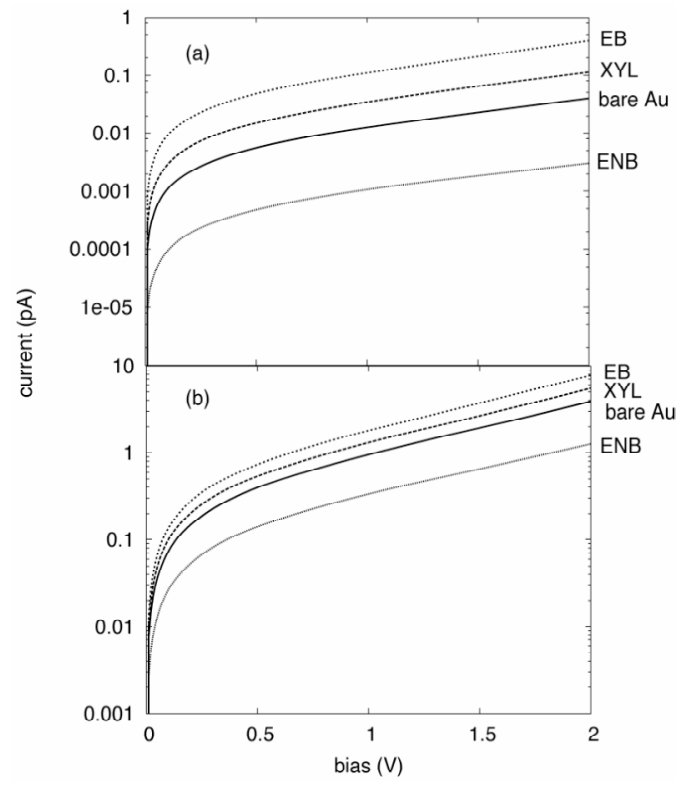

Figure 2. Current-voltage characteristics of the Au(111)-molecule$\mathrm{Au}(111)$ system for the three molecules as well as an empty vacuum gap calculated from (a) equation (5) and (b) equation (6).

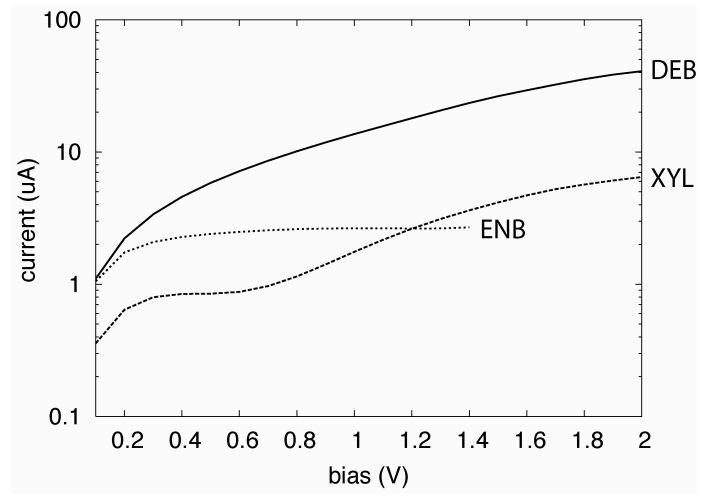

Figure 3. Current-voltage characteristics of 1,4-diethynylbenzene (DEB), 1,4-dimethanethiol (XYL) and 1-ethynyl-4-nitrobenzene adsorbed between $\mathrm{Au}(111)$ electrodes. Calculations were done using standard DFT+NEGF calculations.

The dipole moment of a molecule is therefore potentially an important property with regards to its conductance and current-voltage response. While more complicated mechanisms are clearly present, comparison of the ab-initio results of Fig. 3 with the tunneling barrier model results of Fig. 2 shows that this could be the case for the molecules studied here.

\section{CONCLUSIONS}

We have calculated the tunneling current through different molecules adsorbed between gold slabs using the WKB approximation where the barrier to tunneling is taken to be the work function of gold. We used two methods to calculate the work function. Although they result in different values, the trend is the same: the dipole moment of the gold surface with/without adsorbed molecules affects the work function. Therefore depending on the direction of the dipole of the adsorbed molecule, it can either enhance or impede the tunneling process. Thus the dipole moment may be a very important property when interpreting experiments that probe the conductivity of molecules.

\section{ACKNOWLEDGMENT}

This work was supported by the Australian Research Council and the University of Technology, Sydney. Computational resources were provided under the merit allocation schemes of ac3 (in NSW) and the National Facility, APAC.

\section{REFERENCES}

[1] M. A. Reed, "Molecular-scale electronics," Proc. Ieee, vol. 87, pp. $652-658,1999$

[2] B. Q. Xu and N. J. J. Tao, "Measurement of single-molecule resistance by repeated formation of molecular junctions," Science, vol. 301, pp. 1221-1223, 2003

[3] X. Y. Xiao, B. Q. Xu, and N. J. Tao, "Measurement of single molecule conductance: Benzenedithiol and benzenedimethanethiol," Nano Lett., vol. 4, pp. 267-271, 2004.

[4] J. J. W. M. Rosink, M. A. Blauw, L. J. Geerligs, E. van der Drift, and S. Radelaar, "Tunneling spectroscopy study and modeling of electron 
transport in small conjugated azomethine molecules," Phys. Rev. B, vol. 62, pp. 10459-10466, 2000.

[5] K. Moth-Poulsen, L. Patrone, N. Stuhr-Hansen, J. B. Christensen, J. P. Bourgoin, and T. Bjornholm, "Probing the effects of conjugation path on the electronic transmission through single molecules using scanning tunneling microscopy," Nano Lett., vol. 5, pp. 783-785, 2005.

[6] T. Ishida, W. Mizutani, N. Choi, U. Akiba, M. Fujihira, and H. Tokumoto, "Structural effects on electrical conduction of conjugated molecules studied by scanning tunneling microscopy," J. Phys. Chem. B, vol. 104,pp. 11680-11688, 2000.

[7] M. Dorogi, J. Gomez, R. Osifchin, R. P. Andres, and R. Reifenberger, "Room-temperature coulomb-blockade from a self-assembled molecular nanostructure," Phys. Rev. B, vol. 52, pp. 9071-9077, 1995.

[8] S. Datta, W. D. Tian, S. H. Hong, R. Reifenberger, J. I. Henderson, and C. P. Kubiak, "Current-voltage characteristics of self-assembled monolayers by scanning tunneling microscopy," Phys. Rev. Lett., vol. 79, pp. 2530-2533, 1997.

[9] L. A. Bumm, J. J. Arnold, M. T. Cygan, T. D. Dunbar, T. P. Burgin, L. Jones, D. L. Allara, J. M. Tour, and P. S. Weiss, "Are single molecular wires conducting?," Science, vol. 271, pp. 1705-1707, 1996.

[10] R. P. Andres, T. Bein, M. Dorogi, S. Feng, J. I. Henderson, C. P. Kubiak, W. Mahoney, R. G. Osifchin, and R. Reifenberger, "'coulomb staircase" at room temperature in a self-assembled molecular nanostructure," Science, vol. 272, pp. 1323-1325, 1996.

[11] J. C. Love, L. A. Estroff, J. K. Kriebel, R. G. Nuzzo, and G. M. Whitesides, "Self-assembled monolayers of thiolates on metals as a form of nanotechnology," Chem. Rev., vol. 105, pp. 1103-1169, 2005.

[12] J. Reichert, R. Ochs, D. Beckmann, H. B. Weber, M. Mayor, and H. von Lohneysen, "Driving current through single organic molecules," Phys. Rev. Lett., vol. 88, pp. 176804, 2002.

[13] M. A. Reed, C. Zhou, C. J. Muller, T. P. Burgin, and J. M. Tour, "Conductance of a molecular junction," Science, vol. 278, pp. 252-254, 1997.

[14] T. Bohler, J. Grebing, A. Mayer-Gindner, H. V. Lohneysen, and E. Scheer, "Mechanically controllable break-junctions for use as electrodes for molecular electronics," Nanotechnology, vol. 15, pp. S465-S471, 2004

[15] K. Stokbro, J. Taylor, M. Brandbyge, J. L. Mozos, and P. Ordejon, "Theoretical study of the nonlinear conductance of di-thiol benzene coupled to au(111) surfaces via thiol and thiolate bonds," Comp. Mater. Sci., vol. 27, pp. 151-160, 2003

[16] M. Di Ventra, S. T. Pantelides, and N. D. Lang, "First-principles calculation of transport properties of a molecular device," Phys. Rev. Lett., vol. 84, pp. 979-982, 2000.

[17] X. D. Cui, A. Primak, X. Zarate, J. Tomfohr, O. F. Sankey, A. L. Moore, T. A. Moore, D. Gust, G. Harris, and S. M. Lindsay, "Reproducible measurement of single-molecule conductivity," Science, vol. 294, pp. 571-574, 2001.

[18] Y. Q. Xue and M. A. Ratner, "Microscopic study of electrical transport through individual molecules with metallic contacts. Ii. Effect of the interface structure," Phys. Rev. B, vol. 68, pp. 115407, 2003.

[19] K. H. Muller, "Effect of the atomic configuration of gold electrodes on the electrical conduction of alkanedithiol molecules," Phys. Rev. B, vol. 73, pp. 045403, 2006.

[20] S. H. Ke, H. U. Baranger, and W. T. Yang, "Contact atomic structure and electron transport through molecules," J. Chem. Phys., vol. 122, pp. 074704, 2005

[21] Y. B. Hu, Y. Zhu, H. J. Gao, and H. Guo, "Conductance of an ensemble of molecular wires: A statistical analysis," Phys. Rev. Lett., vol. 95 , pp. 156803, 2005.

[22] R. C. Hoft, J. Liu, M. B. Cortie, and M. Ford, "Electron tunneling through alkanedithiol molecules," presented at Proc. SPIE: BioMEMS and Nanotechnology II, 2006.

[23] H. Basch, R. Cohen, and M. A. Ratner, "Interface geometry and molecular junction conductance: Geometric fluctuation and stochastic switching," Nano Lett., vol. 5, pp. 1668-1675, 2005.

[24] L. J. Sham and W. Kohn, "One-particle properties of an inhomogeneous interacting electron gas," Phys. Rev., vol. 145, pp. 561-567, 1966.

[25] P. Hohenberg and W. Kohn, "Inhomogeneous electron gas," Phys. Rev., vol. 136, pp. B864-B871, 1964.
[26] G. C. Solomon, J. R. Reimers, and N. S. Hush, "Single molecule conductivity: The role of junction-orbital degeneracy in the artificially high currents predicted by ab initio approaches," J. Chem. Phys., vol. 121, pp. 6615-6627, 2004.

[27] P. S. Krstic, D. J. Dean, X. G. Zhang, D. Keffer, Y. S. Leng, P. T. Cummings, and J. C. Wells, "Computational chemistry for molecular electronics," Comp. Mater. Sci., vol. 28, pp. 321-341, 2003.

[28] F. Evers, F. Weigend, and M. Koentopp, "Conductance of molecular wires and transport calculations based on density-functional theory," Phys. Rev. B, vol. 69, pp. 235411, 2004.

[29] A. Bilic, J. R. Reimers, and N. S. Hush, "The structure, energetics, and nature of the chemical bonding of phenylthiol adsorbed on the au(111) surface: Implications for density-functional calculations of molecularelectronic conduction," J. Chem. Phys., vol. 122, pp. 094708, 2005.

[30] J. M. Soler, E. Artacho, J. D. Gale, A. Garcia, J. Junquera, P. Ordejon, and D. Sanchez-Portal, "The siesta method for ab initio order-n materials simulation," J. Phys.-Condens. Mat., vol. 14, pp. 2745-2779, 2002.

[31] P. Ordejon, E. Artacho, and J. M. Soler, "Self-consistent order-n density-functional calculations for very large systems," Phys. Rev. B, vol. 53, pp. 10441-10444, 1996.

[32] N. Troullier and J. L. Martins, "Efficient psudopotentials for planewave calculations," Phys. Rev. B, vol. 43, pp. 1993-2006, 1991.

[33] J. P. Perdew, K. Burke, and M. Ernzerhof, "Generalized gradient approximation made simple," Phys. Rev. Lett., vol. 77, pp. 3865-3868, 1996.

[34] S. Datta, Electronic transport in mesoscopic systems: Cambridge University Press, 1995.

[35] M. B. Cortie, M. H. Zareie, S. R. Ekanayake, and M. J. Ford, "Conduction, storage, and leakage in particle-on-sam nanocapacitors," Ieee T. Nanotechnol., vol. 4, pp. 406-414, 2005.

[36] M. Brandbyge, J. L. Mozos, P. Ordejon, J. Taylor, and K. Stokbro, "Density-functional method for nonequilibrium electron transport," Phys. Rev. B, vol. 65, pp. 165401, 2002.

[37] H. B. Akkerman, P. W. M. Blom, D. M. de Leeuw, and B. de Boer, "Towards molecular electronics with large-area molecular junctions," Nature, vol. 441, pp. 69-72, 2006. 